\title{
Krankheit Intersexualität - Trauma Therapie. Über die medizinische Konstruktion von Geschlecht im Deutschen Ärzteblatt
}

Mareike Kehl

\author{
„it's half biology and half corrective surgery gone wrong \\ you'll notice something funny if you hang around here for too \\ long ago in some black hole before they had these pills to take it back \\ i'm half jill \\ and half jack"1
}

\section{Einleitung}

Melodisch, melancholisch und resigniert singen die „Dresden Dolls“ über die innere Zerrissenheit einer Person, die „half Jill“ und „half Jack“ ist. Über die Bedeutung des Textes schweigt die Sängerin und lässt damit Freiraum zur eigenen Interpretation. ${ }^{2}$ Eine dieser Interpretationen ist im Internet auf der Seite „you tube“ in Form eines Videos zu sehen. ${ }^{3}$ Ist sie die Verarbeitung eines persönlichen Traumas oder eine kritische Auseinandersetzung mit dem Thema Intersexualität? Die Antwort spielt keine Rolle, beinhaltet sie doch in beiden Fällen dieselbe Kernaussage. Es geht um Angst, Ausgrenzung, Scham.

1 Aus „Half Jack“ von den „Dresden Dolls“.

2 Ein Statement der Sängerin zur Interpretation ihrer Liedtexte ist auf der Internetseite der Band zu finden. Vgl. www.dresdendolls.com/contact_theband.htm.

3 In der Beschreibung des Videos wird die Intention klar: „An informative music video of sorts intended to raise awareness and educate others about intersex, focussing specifically on the need to change the current medical model that supports the practice of infant intersex surgery. " Vgl. www.youtube. com/watch?v=IVi5pnCljiU [16.05.2007]. 
Um chirurgische Intervention, um die Bloßstellung vor Medizinstudierenden, um immer wiederholte Untersuchungen durch Ärzte und Ärztinnen, um das Gefühl, anders zu sein. Intersexuelle Menschen tragen oft völlig unterschiedliche medizinische Diagnosen mit sich, eines jedoch ist beinahe allen gemeinsam: Sie sind durch die medizinische „Hölle der Stigmatisierung“" gegangen. ${ }^{4}$ Sie sind traumatisiert.

\subsection{Der therapeutische Grundgedanke}

Das Lindern von Leid und die Schaffung oder Erhaltung von Lebensqualität ist die ethische Grundlage jeden ärztlichen Handelns. Dies gilt auch in Bezug auf die Behandlung von Menschen mit geschlechtlichen Normabweichungen.

Die von der Deutschen Gesellschaft für Kinderchirurgie herausgegebene Leitlinie zur Behandlung von intersexuellen Menschen formuliert als Behandlungsziel eine „Harmonische Identität von genetischem, phänotypischem und soziokulturellem Geschlecht mit Potentia coeundi und generandi soweit als möglich“. ${ }^{5}$ Das Erreichen einer „Beischlaf-“ und „Zeugungsfähigkeit“ soll angestrebt werden. Die Entscheidung über das letztendliche Geschlecht richtet sich nach den chirurgischen Möglichkeiten in Abhängigkeit von den o.g. Grundsätzen. ${ }^{6}$

Im Sinne einer „optimal gender policy “ wird auf eine frühe Geschlechtszuweisung zugunsten einer vermeintlich optimalen psychosexuellen Entwicklung der Kinder gedrängt. ${ }^{7}$ Dabei scheint es ebenso notwendig, den jungen Erwachsenen eine sexuelle Aktivität zu ermöglichen.

Die lange Zeit gängige Praxis aber wird von den „Patienten“ oft als sexualisierte Gewalt, als erniedrigend erlebt und chirurgische Intervention wird keineswegs als „Heilung“ empfunden. ${ }^{8}$ Die Irreversibilität der operativen Maßnahmen und zahlreiche Re-Operationen zur Herstellung möglichst funktionsfähiger äußerer Genitalorgane können zu tiefgreifenden Traumatisierungen führen und stellen die zugrunde liegende Indikation in Frage. ${ }^{9}$ Besonders mit

4 Zu Erlebnissen von Betroffenen mit Ärztinnen und Ärzten und ihren Lebensgeschichten vgl. www.xy-frauen.de und www.beepworld.de/members59/garou68/[Stand: 16.05.2007].

5 "Leitlinien“ sind Hilfen zur ärztlichen Entscheidungsfindung. Sie werden unter Verwendung der aktuellsten wissenschaftlichen Erkenntnisse erstellt und sind rechtlich nicht bindend. Trotzdem orientiert sich die überwiegende Mehrheit der ÄrztInnenschaft an diesen Leitlinien. Vgl. konkret Leitlinie „Intersexualität; Störungen der geschlechtlichen Differenzierung", AWMF-Leitlinien-Register, Nr. 006/105.

6 Vgl. ebd. zur „kontrasexuellen Rekonstruktion (Umwandlung)“. Diese soll angestrebt werden, „wenn auch mit hormonellen und operativen Verfahren eine unzureichende Potenz der Gonade zur Ausprägung eines isosexuell identifizierbaren und akzeptablen genitalen Phänotypus besteht. ,Opferung' einer unzureichenden Potentia generandi zugunsten einer akzeptablen Identität des Phänotypus und der hormonell aufrechterhaltenen Potentia coeundi.“

7 Zurückgehend auf John Money wurde seit 1955 davon ausgegangen, dass eine Geschlechtsidentifikation allein durch Erziehung und Sozialisation bestimmt ist. Money ging von einer geschlechtlichen Neutralität Neugeborener aus und postulierte eine frühe Geschlechtszuweisung innerhalb der ersten Lebensmonate und eine absolute Geheimhaltung gegenüber den Kindern und deren Umwelt. Diese These war lange Zeit Behandlungsrichtlinie und wurde erst 1998 durch Milton Diamond widerlegt. Vgl. hierzu Kipnis/Diamond (1998) und Frewer/Säfken (2005), S. 137-156.

$8 \mathrm{Vgl}$. Fußnote 4.

9 Mit Funktionsfähigkeit ist hier die im Sinne der Leitlinien herzustellende potentia coeundi et generandi gemeint. 
der bei Scheidenplastiken angewandten Methode der Bougierung (Dehnung der Neovagina mittels Metallstäben) assoziieren zahlreiche Frauen übergriffsähnliche Erfahrungen, die ihnen das Erleben einer traumafreien Sexualität verwehren. ${ }^{10}$ Seit den 1990er Jahren wenden sich auch in der Öffentlichkeit Selbsthilfeorganisationen und Zusammenschlüsse von intersexuellen Menschen gegen die Praxis der Geschlechtszuweisung und gegen eine Pathologisierung geschlechtlicher Abweichungen. Eine Auseinandersetzung mit dem Begriff der „Krankheit“ wird gefordert. Gleichzeitig setzt ein Umdenken in der medizinischen Fachwelt ein, das den medizinischen Heilauftrag auch im Sinne der ärztlichen Berufsordnung überdenkt. ${ }^{11}$

Hertha Richter-Appelt misst der „erwünschten oder nicht erwünschten Geschlechtsidentität“ ebenso wie der Geschlechterrolle und der sexuellen Orientierung besondere Bedeutung in der Diagnostik und Behandlung von intersexuellen Menschen bei. ${ }^{12}$ Nach Judith Butlers These, dass ein Zusammenhang zwischen biologischer und körperlicher Geschlechtlichkeit (sex), der psychosozialen Geschlechterrolle (gender) und der Geschlechtsidentität in Abhängigkeit von kulturellen und gesellschaftspolitischen Einflüssen einer fortwährenden Veränderung unterliegt, wäre zu fordern, dass auch eine medizinische Sichtweise dieser Veränderung Rechnung trägt. ${ }^{13}$ „Erwünschte“ oder „nicht erwünschte“ Geschlechtsidentitäten sind demnach gesellschaftlich konstruiert. Innerhalb des seit 2002 DFG-geförderten Netzwerk Intersexualität entwickelt die Arbeitsgruppe Medizinethik und Intersexualität um Claudia Wiesemann ethische Crundsätze und Empfehlungen zum therapeutischen Umgang mit Besonderheiten der Geschlechtsentwicklung unter Einbeziehung von Eltern und Betroffenen. ${ }^{14}$ In Abgrenzung zu Erkrankungen, die als Folge einer untypischen Entwicklung der Geschlechtsorgane durchaus eine medizinische Intervention erfordern, wie etwa assoziierte Fehlbildungen anderer Organe oder Störungen der Steroidhormonproduktion, stellt die Arbeitsgruppe die ethischen Aspekte in den Vordergrund. Sie erkennt einen „kulturellen Wandlungsprozess“, der

„gekennzeichnet ist durch ein allmähliches Aufbrechen geschlechtlicher Normen sowie eine größere Toleranz für geschlechtliche Uneindeutigkeit bei Männern und Frauen. Vor dem Hintergrund der früheren Tabuisierung dieses Themas und der bis heute anhaltenden Stigmatisierung von Betroffenen sollen diese Grundsätze und Empfehlungen einen professio-

10 Die in den Leitlinien implizierten Intentionen der Normierung des Körpers von Intersexuellen zugunsten einer Normalisierung ihrer geschlechtsspezifischen Sozialisation werden von den Berichten Betroffener eines Besseren belehrt. Die vorgebliche Notwendigkeit der Regulierung des Körpers zum Erreichen einer Beischlaf- und Zeugungsfähigkeit führt allzu oft zu einer sexuellen Traumatisierung.

11 Neben verschiedenen grundlagenorientierten Forschungsprojekten, unter anderem in DFG-geförderten Forschungsprojekten an den Universitätskliniken Hamburg und Lübeck, beschäftigen sich eine Reihe WissenschaftlerInnen aus unterschiedlichen Disziplinen auch mit ethischen Aspekten der (Behandlung von) Intersexualität. Vgl. www.forschergruppe-is.uk-sh.de und www.netzwerk-is.de.

12 Vgl. Richter-Appelt (2007), S. 53.

13 Vgl. Butler (1991).

14 Die Empfehlungen wurden im Herbst 2006 von der AG verabschiedet und sind auf der Internetseite des Netzwerkes im Forum zu Diskussion gestellt. Vgl. www.netzwerk.-is.de. 
nellen, respektvollen und toleranten Umgang mit DSD fördern und einem stigmatisierenden Verhalten von Medizin und Gesellschaft entgegenwirken. ${ }^{\text {“15 }}$

In den Empfehlungen, die bisher die Leitlinien der Deutschen Gesellschaft für Kinderchirurgie an offizieller Stelle noch nicht abgelöst haben, heißt es außerdem:

„Konflikte entstehen durch das Fehlen eines sozialen Raums, in dem Kinder mit DSD ihr Anderssein als normal erleben können. “16

Dazu gehört, dass auch die Eltern durch das vermeintliche Anderssein ihrer Kinder stark verunsichert werden. So ist selbst der kleinste soziale Raum, eine vertrauensvolle Eltern-Kind-Beziehung, von einem gesellschaftlichen Druck zur frühestmöglichen chirurgischen Geschlechtszuweisung geprägt.

Im Rahmen einer ersten Katamnese-Studie zur Art und Weise sowie zum angemessenen Zeitpunkt einer geschlechtszuweisenden Operation zeigt Richter-Appelt auf, dass besonders das Paradigma der Geheimhaltung gegenüber der Umwelt eine starke Belastung für die Betroffenen und die Eltern-KindBeziehung darstellt. ${ }^{17}$

Die Entscheidung über invasive Maßnahmen ohne die Einbeziehung der Kinder stellt einen Übergriff und widerspricht dem $\$ 7$ der Berufsordnung für Ärzte. Hier heißt es zu Behandlungsgrundsätzen und Verhaltensregeln:

„(1) Jede medizinische Behandlung hat unter Wahrung der Menschenwürde und unter Achtung der Persönlichkeit, des Willens und der Rechte, insbesondere des Selbstbestimmungsrechts von Patientinnen bzw. Patienten zu erfolgen. “18

Für eine altersspezifische Unterstützung des Kindes muss auch eine hinreichende Aufklärung der Eltern gewährleistet sein. Eine Unterscheidung zwischen Elternwunsch und kindlichen Bedürfnissen ist zwingend notwendig. Um dem Kind ein Heranwachsen ohne Stigmatisierungen und Traumatisierung zu ermöglichen, ist eine professionelle psychologische Betreuung der Eltern von Nöten, die eine Akzeptanz des Kindes ermöglichen muss.

\subsection{Fragestellung}

Durchaus können wir also von einem gewissen Paradigmenwandel bezüglich des medizinisch empfohlenen Umgangs mit intersexuellen Kindern sprechen. Nun ist es mitunter ein weiter Weg zwischen der Änderung einer Empfehlung und einer Änderung im konkreten medizinischen Handeln. Die (medizinische) Wissensproduktion spielt in diesen Entwicklungen eine wichtige Rolle. ${ }^{19}$ In-

15 Ebd., der medizinische Ausdruck DSD (disorders of sex development) wird übersetzt mit „Besonderheiten in der Geschlechtsentwicklung“ (Intersexualität).

16 Ebd.

17 Vgl Richter-Appelt (2004).

18 Vgl. Berufsordnung für die nordrheinischen ÄrztInnen in der Fassung vom 20.05.2005, S. 8.

19 Mit Wissen ist nicht etwa ,richtiges ‘ Wissen gemeint. Vielmehr beinhaltet dieser Begriff auch Vorurteile, Fehleinschätzungen, Empfindungen etc. 
wieweit das Wissen innerhalb des medizinischen Diskurses Ärztinnen und Ärzte außerhalb einer Fachelite erreicht, welche jeweils gültigen Wahrheiten vermittelt werden, zeigt eine Untersuchung der Ausgaben des Deutschen Ärzteblattes der letzten zehn Jahre. Der Untersuchung liegt die Methode der Kritischen Diskursanalyse zugrunde, die Siegfried Jäger im Anschluss an Foucault entwickelte. ${ }^{20}$ Die kritische Komponente liegt bereits in der Erfassung des Diskurses,

„indem dabei die implizierten und nicht gesagten Voraussetzungen und als Wahrheiten vertretenen Setzungen oder zu unrecht Konsens beanspruchenden Aussagen oder falsche Verallgemeinerungen und dementsprechenden Fluchtlinien etc. sichtbar gemacht werden können. “21

Die Analyse macht deutlich, für welche „Wahrheiten“ im Deutschen Ärzteblatt Akzeptanz geschaffen wird und zeigtkonkret, welche gesellschaftspolitischen Annahmen einer geschlechtsspezifischen Kategorisierung zugrunde liegen.

\section{Intersexualität im Spiegel des „Deutschen Ärzteblatts“}

Das Deutsche Ärzteblatt (DÄ) wird von der Bundesärztekammer und der kassenärztlichen Bundesvereinigung herausgegeben und erreicht als offizielles Organ der ÄrztInnenschaft alle bei der Bundesärztekammer gemeldeten Ärztinnen und Ärzte. Nach eigenen Angaben ist das DÄ „der mit Abstand meistgelesene Titel der gesamten ärztlichen Fachpresse“. ${ }^{22}$ Neben der im Inhaltsverzeichnis optisch hervorgehobenen medizinisch-wissenschaftlichen Rubrik „Medizin“ gibt es Rubriken mit gesundheits- und sozialpolitisch ausgerichteten Themen, wie „Politik“, „Kultur“ und „Themen der Zeit“. Ein Archiv mit den Volltexten aller in der Printausgabe erschienenen Artikel seit Januar 1996 steht auf der Internetseite frei zur Verfügung. ${ }^{23}$

Im Zeitraum von 1996 bis zur Ausgabe 18/2007 erschienen nur wenige Artikel zur Thematik Intersexualität. Von den relevanten vier Artikeln erschienen zwei unter der Rubrik „Medizin“, zwei weitere Artikel sind in Form von LeserInnenbriefen in der Rubrik „Diskussion“ $z$ u finden. ${ }^{24}$

\footnotetext{
20 Konkret zur Methodik vgl. Jäger (2004).

21 läger (2004), S. 223.

22 Vgl. www.aerzteblatt.de/v4/mediadaten/md_home.asp.

23 Vgl. www.aerzteblatt.de/v4/archiv/simplemask.asp.

24 Zusätzlich zu den hier behandelten Artikeln fanden sich einige weitere, die aber jeweils unterschiedliche Erscheinungsformen der Intersexualität nur im Rahmen von Grundlagen zu diskutierten Krankheitsbildern erwähnten und nicht weiter darauf eingingen.
} 


\subsection{Implikate und Argumentationsstrategien: Androgenresistenz und "unerwünschte Virilisierung“}

„Klinische und molekulare Grundlagen“"25 des Androgenresistenzsyndroms sind das Hauptthema eines von einer Arbeitsgruppe um Olaf Hiort, Paul-Martin Holterhus und Gernot Sinnecker an den Kinderkliniken der Universität Lübeck und des Klinikums Wolfsburg veröffentlichten Beitrags vom März 1999. Hiort ist Stellvertretender Vorsitzender des Netzwerks Intersexualität und war federführend an einem 2006 ausgelaufenen DFG-Forschungsprojekt der Interdisziplinären klinischen Forschergruppe Intersexualität beteiligt ${ }^{26}{ }^{2}$ Holterhus ist ebenfalls Mitglied des Netzwerks Intersexualität und jetziger Leiter des pädiatrisch-endokrinologischen Labors der Kinderklinik an der Universität Kiel. Sinnecker ist Mitglied der Arbeitsgemeinschaft Kinder- und Jugendgynäkologie e.V. und Leiter der Kinderklinik Wolfsburg. Kruse war bis 2001 Leiter der Universitätskinderklinik in Lübeck.

Der Artikel befasst sich mit Defekten des Enzyms 5 a-Reduktase, das die Umwandlung des im Hoden produzierten Testosterons in das wirksamere und für eine männliche Geschlechtsentwicklung notwendige Dihydrotestosteron katalysiert. Darüber hinaus wird auf Mutationen des Androgenrezeptors eingegangen, welcher die Wirkungen von Testosteron und Dihydrotestosteron vermittelt. Grundlagen der Biosynthese von Androgenen allgemein und die intrauterine und pubertäre Wirkung von Hormonen auf die Geschlechtsentwicklung bilden den Einstieg in das Thema. Weiterhin gehen die Autoren auf die endokrinologische und molekulargenetische Diagnostik bei intersexuell geborenen Kindern ein.

Die Veröffentlichung des Artikels unter der Rubrik „Medizin“ und der Titel des Artikels lassen eine medizinisch-wissenschaftliche Herangehensweise an die komplexe Thematik Intersexualität erwarten. Dass dem Thema aber, innerhalb des medizinisch-naturwissenschaftlichem Diskurses, auch gesellschaftliche Implikate zugrunde liegen, zeigt die genauere Durchsicht des Artikels.

So geht es nicht nur um Endokrinologie und Molekularbiologie: Es ist für medizinisch vorgebildete LeserInnen sicherlich nachvollziehbar, dass eine Salzverlustkrise beim Adrenogenitalen Syndrom eine Lebensbedrohung und damit eine Notfallsituation darstellen kann. In derselben Argumentationslinie, also im Kontext des Notfalls, wird hier allerdings auch vertreten, dass „Ursachen einer isolierten gestörten Geschlechtsentwicklung“ einer „raschen Abklärung" bedürften, denn

„Die Unsicherheit hinsichtlich der Geschlechtszugehörigkeit des Kindes führt zu einer außerordentlichen psychischen Belastung für die Eltern“ ${ }^{27}$

Raum für die Kinder bleibt hier nicht, die elterlichen Bedürfnisse werden in den Vordergrund gerückt und mit der Notwendigkeit einer raschen Diagnose

25 Vgl. Hiort et al. (1999a).

26 www.forschergruppe-is.uk-sh.de/index.htm.

27 Vgl. Hiort et al. (1999a). 
und Therapie verkoppelt. Dass es sich dabei explizit um die Situation der unsicheren Einordnung in das binäre System von Mann und Frau handelt, wird ersichtlich, wenn vom Zeitpunkt der „klinischen Auffälligkeit“ die Rede ist. Primäre Amenorrhö, Infertilität oder eine fehlende Pubertätsentwicklung werden als Ursachen für die Konsultation von FachärztInnen und als Symptom einer Androgenresistenz bei phänotypisch eindeutigen Frauen oder Männern beschrieben: „Patienten mit intersexuellem Genitale hingegen stellen einen pädiatrischen Notfall dar." 28

In der Argumentation für die klare Zuordnung zu einem Geschlecht wechseln sich naturwissenschaftliche und gesellschaftspolitische Aussagen ab. So wird der Ursache der pubertären Virilisierung des Genitales bei Kindern mit 5 a-Reduktase-Mangel molekulargenetisch und endokrinologisch nachgegangen, gleichzeitig jedoch deutlich gemacht, dass bei

„einem Kind mit männlichem Kerngeschlecht, aber weiblicher Geschlechtszuordnung [...] vor dem Pubertätsalter eine operative Entfernung der Gonaden erfolgen [sollte], um eine unerwünschte Virilisierung während der Pubertät zu vermeiden. ${ }^{\text {“29 }}$

Wer die Virilisierung als „unerwünscht“ erachtet, bleibt offen. Lediglich eine Wiederholung der Aussage im Zusammenhang mit der Androgenrezeptorresistenz ist zu finden. Hier wird die Art der Virilisierung konkretisiert. Sie wird als „Stimmvertiefung und Klitorishypertrophie“ zur Rechtfertigung für die Empfehlung einer Gonadektomie..$^{30}$

Die Autoren vermitteln keinesfalls eine ausschließlich medizinisch begründete Auseinandersetzung mit dem Thema Intersexualität, die eine Einordnung in einen gesellschaftlichen Kontext offen ließe, sondern setzen klar die Notwendigkeit einer Anpassung von biologischen Tatsachen an eine bestehende gesellschaftliche Norm voraus. ${ }^{31}$ Die Verknüpfung von gesellschaftspolitischen Aussagen und Krankheitsbegriffen offenbart das Festhalten am Status quo und unterstellt die Überwindbarkeit der sozialen Ausgrenzung durch medizinische Intervention. ${ }^{32}$

\subsection{Kritisches Hinterfragen}

Der Artikel von Hiort et al. (1999a) zeigt deutlich, inwiefern sich diskursive Konstruktionen von Geschlechtsidentität innerhalb des medizinischen Diskurses in einem binären Rahmen bewegen. Durch gesellschaftspolitische

28 Ebd.

29 Ebd.

30 Ebd.

31 Dabei unterstreicht das Spezialwissen der Autoren deren wissenschaftliche Autorität und unterstützt die Aussage, die vorgelegten Forschungsergebnisse seien ein „wichtiger Beitrag für die Differentialdiagnose bei intersexuellem Genitale und die medizinische Betreuung betroffener Patienten“. Als Referenz führen sich die Autoren selbst an.

32 Dass der medizinische wie auch der gesamtgesellschaftliche Diskurs aber durchaus wandelbar ist und dabei auch kulturellen und gesellschaftlichen Einflüssen unterliegt, zeigt sich am Beispiel der Homosexualität. Noch im frühen 20. Jahrhundert galt gleichgeschlechtliche Liebe als Krankheit. 1991 wurde Homosexualität aus dem ICD-10 gestrichen und erfährt heute eine zunehmende gesellschaftliche Akzeptanz. 
Aussagen wird die scheinbare Notwendigkeit medizinischer Interventionen als unumstößliche Wahrheit gefestigt, und es wird zumindest im DÄ dazu beigetragen, dass diese Wahrheit gesellschaftsfähig bleibt.

Ohne Reaktion bleibt der Artikel allerdings nicht. Im Juli 1999 veröffentlichte das DÄ einen Leserbrief von Dr. med. Hartmut Cardeneo zum Beitrag von Hiort et al. „Verdienstvoll“" sei die Zusammenstellung gewesen, betont Cardeneo zu Beginn, ihn beunruhige jedoch „dass gleich zweimal [...] die Gonadektomie empfohlen wird, damit fälschlich als Mädchen eingestufte Kinder auch Mädchen bleiben müssen. ${ }^{\text {"33 }}$ Cardeneo kritisiert hier eine operative Fixierung des den Kindern zugedachten Geschlechts und stellt die Richtigkeit dieser Zuordnung in Frage, wobei hier offengelassen wird, welche und wie viele unterschiedliche Geschlechterrollen neben der weiblichen noch zu finden sind. Männlich und Weiblich werden nicht starr gegenübergestellt. Eine Betonung einer auferlegten und nicht von Kindern selbst entschiedenen Geschlechterrolle findet sich in der Bezeichnung, die Cardeneo für das therapeutische Team wählt:

„Von wem ist ,die Virilisierung unerwünscht'?, von der Administration, oder von den Eltern? $?^{\prime 34}$

Der Begriff „Administration“ verweist auf das Fehlen einer persönlichen, menschlichen Ebene und hinterfragt provokant den ärztlichen „Heilungsauftrag“. Die Dringlichkeit einer Einbeziehung der PatientInnen in die ärztliche und elterliche Entscheidungsfindung begründet Cardeneo auch mit rechtlichen Konsequenzen. ${ }^{35}$

Eine klare Argumentationsstrategie ist im Leserbrief nicht zu finden, die Aneinanderreihung von verschiedenen Aspekten und gesellschaftspolitischen Grundannahmen bestärkt allerdings die kontroverse Diskussion um klare Behandlungsleitlinien. Anstatt der Forschungsgruppe Alternativen entgegenzusetzen, beschränkt sich der Autor hier auf das provozierende und kritische Hinterfragen.

Eine wichtige Schlussfolgerung zieht der Autor aus den unbekannten Auswirkungen von Androgenen auf die Entwicklung einer Geschlechtsidentität auch im ZNS:

„Dieses Wissen [der hormonellen Einflüsse auf die Entwicklung der Geschlechterrolle im ZNS, M. K.] wäre aber unbedingte Voraussetzung im Sinne des nihil nocere, im Kindesalter Festlegungen der Geschlechtsrolle an nicht einwilligungsfähigen Menschen vorzunehmen. Damit aus einem Inter-Sex nicht völlig unnötig ein Null-Sex gemacht wird. ${ }^{436}$

Cardeneo stellt damit indirekt das in den Leitlinien der Deutschen Gesellschaft für Kinderchirurgie formulierte therapeutische Ziel in Frage. Er konstatiert das

33 Vgl. Cardeneo (1999).

34 Ebd.

35 Die Verschränkungen mit dem juristischen Diskurs um Intersexualität sollen hier nicht näher untersucht werden. Es lohnt sich aber eine genauere Untersuchung der Auswirkungen der zivil- und strafrechtlichen Situation auf den Gesamtdiskurs um Intersexualität.

36 Vgl. Cardeneo (1999). 
(unnötige) Verhindern einer sexuellen Erlebnisfähigkeit durch medizinische Intervention. Unterstützend zieht Cardeneo Parallelen zu Beschneidungen an Mädchen in Afrika und bringt damit die Thematik der Menschenrechte mit ein. ${ }^{37}$ Dabei geht es ihm um die Folgen des Eingriffs, der „potentiell geeignet ist, sexuelles Erleben vollständig zu unterdrücken. " ${ }^{8}$

\subsection{Festschreiben von Wahrheiten}

In einer in derselben Ausgabe des DÄ veröffentlichten Replik der Arbeitsgruppe von Hiort et al. auf den Leserbrief Cardeneos werden Implikate aus deren ursprünglichem Beitrag wiederholt und vertieft. Zunächst festigen die Autoren ihre Autorität, indem sie einerseits die ärztliche Kompetenz Cardeneos als Kollegen betonen, andererseits aber ihre eigenen Untersuchungen und ihre intensiven Beschäftigungen mit der Thematik als Referenz anführen. Zudem machen sie deutlich, dass die dann folgenden Erläuterungen bereits in ihrem Artikel erarbeitet wurden, demnach also als bekannt und verstanden vorausgesetzt werden.

Da der phänotypische Aspekt vieler Kinder mit 5 a-Reduktase-Mangel „komplett oder fast komplett weiblich“ imponiert, „werden diese Kinder [...] als Mädchen aufgezogen“. ${ }^{39}$ Sie „sollten während der Kindheit eine möglichst normale Geschlechtsrollenentwicklung erleben“. „Normal“ wird in diesem Zusammenhang gleichgesetzt mit „weiblich normal“, eine männliche oder gar individuelle Entwicklung ist nicht vorgesehen und eine Virilisierung sei

„tatsächlich ein sehr unerwünschter Effekt, da dies neben den körperlichen Merkmalen zu schweren Identitätskrisen führen kann. Daher empfehlen wir unbedingt die Gonadektomie vor Pubertätsbeginn, um diese dem Phänotyp und damit der Geschlechtsidentität entgegenstehenden Wirkungen zu vermeiden. ${ }^{40}$

Die Schlussfolgerung ist interessant. Ein weibliches Äußeres muss also zwangsweise zu einer weiblichen Geschlechtsidentität geführt werden. Die Ursache der Identitätskrisen liegt nach Hiort et al. ausschließlich in der Virilisierung und der damit verbundenen gesellschaftlichen Ausgrenzung innerhalb eines binären Geschlechtermodells. Dieses wird a priori angenommen und keinesfalls hinterfragt.

Allein „chirurgische, kulturelle und psychische Einflussfaktoren“ müssen neben diagnostischen Möglichkeiten bei der Geschlechtszuordnung von Kindern mit intersexuellen Genitalien mitberücksichtigt werden. ${ }^{41}$ Inwiefern es also einer gewissen Willkür, die auch von der chirurgischen „Machbarkeit“ abhängt, unterliegt, welchem Geschlecht ein Kind zugeordnet wird, sei in den

37 Auch hier ist eine Diskursverschränkung zu verzeichnen, der nachgegangen werden sollte. Es gibt mittlerweile auch ärztliche Zusammenschlüsse, die gegen Beschneidungen von Mädchen und Frauen mobilisieren. Diese beziehen eindeutig Position gegen menschenunwürdige Eingriffe in das Sexualleben der Betroffenen.

38 Vgl. Cardeneo (1999).

39 Vgl. Hiort et al. (1999b).

40 Ebd.

41 Ebd. 
Raum gestellt und erinnert an die von John Money postulierten und stark kritisierten Ansätze zur geschlechtlichen Neutralität Neugeborener. Immerhin räumen die Autoren ein, dass allgemeingültige Vorgehensweisen schwer zu formulieren seien, konstatieren jedoch:

„In unserem Kulturkreis ist aber eine eindeutige Geschlechtszuordnung aus rechtlichen, soziologischen und psychologischen Gründen unbedingt vonnöten. Daher sollte hier eine Korrekturoperation des äußeren Genitale so frühzeitig erfolgen, daß sich das Kind im Rahmen seiner Entwicklung eindeutig einem Geschlecht und dessen Erlebnisfähigkeit zuordnen kann." ${ }^{42}$

Für die geschlechtsassoziierte Erlebnisfähigkeit der Kinder ist es im Sinne der Autoren unabdingbar, eindeutige äußere Geschlechtsmerkmale operativ herzustellen. Dass diese Operationen dazu beitragen, eine bessere Identifikation mit der Geschlechterrolle zu schaffen, ist fraglich. Äußerungen von Betroffenen lassen zum Teil tiefe Traumatisierungen durch chirurgische Eingriffe erahnen, die sich auch auf eine sexuelle Erlebnisfähigkeit auswirken. Denn diese ist ja gerade nicht nur auf ein körperliches Empfinden reduziert, sondern eng verbunden mit einem positiven Körpergefühl.

Doch kritisieren die Autoren gerade im Zusammenhang mit dem Erhalt einer sexuellen Erlebnisfähigkeit Cardeneos Vergleich mit Beschneidungen junger Mädchen. Cardeneo ging es hier allerdings um die Folgen eines derartigen Eingriffes in die körperliche Integrität der Betroffenen, nicht um dessen Intention.

In der Replik wird noch mehr als im ursprünglichen Artikel eine gesellschaftspolitische Stellungnahme deutlich, welche die unbedingte Zuordnung eines betroffenen Kindes zu einem Geschlecht innerhalb der normativen Geschlechterordnung als unveränderliche Wahrheit darstellt.

\subsection{Kein Paradigmenwechsel im Deutschen Ärzteblatt}

Zwischen 1999 und 2005 erschien im DÄ kein weiterer Artikel zur Intersexualität. Der dann veröffentlichte Beitrag im März 2005 ist ebenfalls von einer Mitarbeiterin des BMBF-geförderten Netzwerks Intersexualität verfasst. Annette Richter-Unruh war bis zum Sommer 2006 Funktionsoberärztin an der Universitätskinderklinik Essen und arbeitete gemeinsam mit Olaf Hiort und PaulMartin Holterhus an einer Studie zu klinischen Auswirkungen und molekularen Grundlagen der Androgenbiosynthese.

Der Artikel behandelt wie der Beitrag von Hiort et al. (1999a) molekulargenetische und hormonelle Grundlagen der Geschlechtsdifferenzierung, hier bezogen auf Mutationen im LH-Rezeptor-Gen. Dabei wird besonderes Augenmerk auf die durch inaktivierende Mutationen entstehende Leydigzellhypoplasie als Ursache für primäre Amenorrhö und Infertilität bei Frauen und Hypospadien und intersexuelles Genitale bei Männern gelegt. Der Artikel ist ebenfalls unter der Rubrik „Medizin“ im DÄ veröffentlicht.

42 Ebd. 
Richter-Unruhs Ausführungen haben überwiegend deskriptiven Charakter, gesellschaftspolitisch relevante Äußerungen finden sich kaum. Nur im Zusammenhang mit einem hypergonadotropen Hypogonadismus bei der „milden Form “ der Leydigzellhypoplasie postuliert die Autorin eine operative Korrektur der äußeren Genitale:

„Bei postpartal sehr kleinem Penis kann ein gutes Peniswachstum durch eine dihydrotestosteronhaltigen Salbe erreicht werden. Je nach Ausmaß der Hypospadie muss diese operativ korrigiert werden“. ${ }^{43}$

Inwiefern es der Autorin hier um einen kosmetischen Eingriff geht, der eine Bedeutsamkeit der Penisgröße für die Geschlechtsidentität voraussetzen müsste, bleibt offen. Weitere Aussagen, insbesondere in Bezug auf eine geschlechtsassoziierte Entwicklung der Kinder, finden sich nicht.

Bei Mädchen und Frauen mit einem LH-Rezeptordefekt und männlichem Karyotyp (46, XY)

„wird die Entfernung der Gonaden aufgrund eines möglichen Entartungsrisikos analog den Patientinnen mit gemischter Gonadendysgenesie empfohlen. ${ }^{\text {"44 }}$

Hier ist eine andere Herangehensweise zu bemerken. War es 1999 noch ein „Muss“, die Gonaden zu entfernen, und zwar aufgrund einer möglichen „unerwünschten Virilisierung“, so wird nun eine Entfernung der Gonaden den Patientinnen nur nahegelegt, nicht aber als unabdingbar vorausgesetzt. Dass hier ein Vergleich dieser beiden Aussagen nur eingeschränkt zulässig ist, sei zu erwähnen, da aufgrund der unterschiedlichen zugrunde liegenden hormonellen Dysregulationen andere therapeutische Konsequenzen zu ziehen sind. Dennoch ergibt sich das Bild einer differenzierteren Herangehensweise. Ein kritisches Hinterfragen der gesellschaftspolitischen Ansätze ist allerdings auch hier nicht zu finden.

\section{Resümee}

Die körperliche und psychische Integrität intersexueller Kinder ist sowohl durch die medizinischen Behandlungsmaßnahmen als auch durch gesellschaftliche Normvorstellungen extrem gefährdet.

Im DÄ wird überwiegend das Bild eines absoluten Imperativs zum operativen Eingriff in den Körper intersexueller Kinder vermittelt. Gerade die Ausführungen von Hiort et al. vermitteln die unbedingte Notwendigkeit der Anpassung an gesellschaftliche Gegebenheiten. Diese Aussage wird allerdings keinesfalls als diskussionswürdige Annahme gekennzeichnet und somit eben auch nicht zur Diskussion gestellt. Die Autoren erwecken vielmehr den Schein einer rein naturwissenschaftlichen Ausführung und entziehen sich damit jeglicher gesellschaftlicher Kontroverse - und das bei einem gesellschaftspolitisch durchaus umkämpften Feld. Die kritische Antwort auf diesen Artikel hinter-

43 Vgl. Richter-Unruh (2005).

44 Ebd. 
fragt genau die als medizinische Feststellungen ausgegebenen gesellschaftlichen Aussagen. Mit den Artikeln über Intersexualität trägt das DÄ nicht zur notwendigen Diskussion über die Implikate der medizinischen Verfahrensweise mit Intersexuellen bei, vielmehr werden bestehende Vorannahmen festgeschrieben. Dem Paradigmenwechsel wird nicht Rechnung getragen.

Erstaunlich ist, wie viele gesellschaftliche Festschreibungen die medizinische Fachliteratur vermittelt. Die Herstellung klarer Verhältnisse innerhalb einer zweigeschlechtlichen Ordnung bleibt als Ziel bestehen. Eltern wollen und sollen ihr Kind als Sohn oder Tochter erziehen. Die elterliche Unsicherheit im Umgang mit ihren intersexuellen Kindern wird in den Vordergrund gerückt. Diese Eltern werden so zu Patienten. Die medizinische Maßnahme an den Kindern wird (auch) mit einer psychischen Belastung für die Eltern begründet.

Andererseits wird der Eingriff in die persönliche körperliche und psychische Integrität der Betroffenen damit begründet, dass die Entwicklung einer Geschlechtsidentität nur durch eine exakte Identifizierung mit der männlichen oder weiblichen Geschlechterrolle möglich sei. Eine Geschlechtsidentität losgelöst von der normativen Kategorisierung innerhalb eines binären Systems wird von vornherein ausgeschlossen.

Das DÄ ist die einzige medizinische Fachzeitschrift, die alle bei der Bundesärztekammer gemeldeten Ärztinnen und Ärzte erreicht. Umso mehr erstaunt es, dass einem sich abzeichnenden Paradigmenwechsel in der Behandlung von Menschen mit Abweichungen in der Geschlechtsentwicklung hier nicht Rechnung getragen wird, mehr noch, dass innerhalb eines so kontrovers diskutierten Themas gesellschaftspolitische Aussagen unhinterfragt als „Wahrheiten" beschrieben werden.

Wie die übrige Fachpresse das Thema verhandelt, wie sich dieser Spezialdiskurs auf den gesamtgesellschaftlichen Diskurs auswirkt, soll in weiteren Analysen untersucht werden.

\section{Literatur}

Ärztekammer Nordrhein (2005): Ärztekammer Nordrhein (Hrsg.), Berufsordnung für die nordrheinischen Ärztinnen und Ärzte (2005)

Butler (1991): Judith Butler, Das Unbehagen der Geschlechter, Frankfurt a. M. 1991

Cardeneo (1999): Hartmut Cardeneo, Androgenresistenzsyndrome - Klinische und molekulare Grundlagen: Nicht die Geschlechterrolle festlegen, Deutsches Ärzteblatt 96 (1999), 27, S. A1845 f.

Kipnis/Diamond (1998): Kenneth Kipnis, Milton Diamond, Pediatric Ethics and the Surgical Assignment of Sex, The Journal of Clinical Ethics 9 (1998), 4, p. 398-410

Frewer/Säfken (2005): Andreas Frewer, Christian Säfken, Identität, Intersexualität, Transsexualität: Medizinhistorische und ethisch-rechtliche Aspekte der Geschlechtsumwandlung, in: Frank Stahnisch, Florian Steger (Hrsg.): Medizin, Geschichte und Geschlecht. Körperhistorische Rekonstruktionen von Identitäten und Differenzen, Stuttgart 2005, S. 137-156

Hiort et al. (1999 a): Olaf Hiort, Paul-Martin Holterhus, Gernot H. G. Sinnecker et al., Androgenresistenzsyndrome - Klinische und molekulare Grundlagen, Deutsches Ärzteblatt 96 (1999), 11, S. A686-693

Hiort et al. (1999 b): Olaf Hiort, Paul-Martin Holterhus, Gernot H. G. Sinnecker et al., Androgenresistenzsyndrome - Klinische und molekulare Grundlagen: Schlusswort, Deutsches Ärzteblatt 96 (1999), 27, S. A1846 
läger (2004): Siegfried läger, Kritische Diskursanalyse, Münster, 4. Aufl., 2004

Richter-Appelt (2004): Hertha Richter-Appelt, Intersexualität und Medizin - Erste Ergebnisse eines Forschungsprojektes, Zeitschrift für Sexualforschung 17 (2004), S. 239-257

Richter-Appelt (2007): Hertha Richter-Appelt, Intersexualität - Störungen in der Geschlechtsentwicklung, Bundesgesundheitsblatt - Gesundheitsforschung - Gesundheitsschutz 50 (2007), S. 52-61

Richter-Unruh (2005): Annette Richter-Unruh, Leydigzell-Hypoplasie und Testotoxikose - wenig bekannte Krankheitsbilder: Klinische und molekulare Grundlagen bei Vorliegen von Mutationen im LH-RezeptorGen, Deutsches Ärzteblatt 102 (2005), 10, S. A673-678

\section{Internet}

www.aerzteblatt.de/v4/archiv/simplemask.asp (Archiv des Deutschen Ärzteblatts)

www.awmf.org (Leitlinie „Intersexualiät; Störungen der geschlechtlichen Differenzierung“, AWMF-LeitlinienRegister, Nr. 006/105)

www.beepworld.de/members59/garou68/(Erfahrungsbericht eines Betroffenen)

www.dresdendolls.com/contact_theband.htm (Band „The Dresden Dolls“)

www.forschergruppe-is-sh.de (Forschergruppe Intersexualität)

www.netzwerk-is.de (Netzwerk Intersexualität)

www.youtube.com/watch?v=IVi5pnCljiU (Video „Half Jack“)

www.xy-frauen.de (Selbsthilfeorganisation xy-Frauen) 